\title{
What Injury Mechanism and Patterns of Ligament Status Are Associated With Isolated Coronoid, Isolated Radial Head, and Combined Fractures?
}

\author{
In Hyeok Rhyou MD, Ji-Ho Lee MD, Kyung Chul Kim MD, \\ Kee Baek Ahn MD, Seong Cheol Moon MD, Hyeong Jin Kim MD, \\ Jung Hyun Lee MD
}

Received: 13 December 2016/Accepted: 5 April 2017/Published online: 12 April 2017

(C) The Association of Bone and Joint Surgeons (R 2017

\begin{abstract}
Background Isolated coronoid, isolated radial head, and combined coronoid and radial head fractures are common elbow fractures, and specific ligamentous injury of each fracture configuration has been reported. However, the osseous injury mechanism related to ligament status remains unclear.

Questions/purposes The objectives of this study were: (1) to determine what ligamentous injury patterns (medial or lateral collateral) and bone contusion patterns (medial or lateral) are associated with isolated coronoid, isolated radial head, and combined coronoid and radial head fractures; (2) to correlate the osseous injury mechanism based on these findings with isolated coronoid, isolated radial head, and combined coronoid and radial head fractures; and (3) to determine whether isolated and combined coronoid fractures have different fracture lines through the coronoid (tip or anteromedial facet), speculated to be caused by different injury mechanisms.
\end{abstract}

Each author certifies that he or she has no commercial associations (eg, consultancies, stock ownership, equity interest, patent/licensing arrangements, etc) that might pose a conflict of interest in connection with the submitted article.

All ICMJE Conflict of Interest Forms for authors and Clinical Orthopaedics and Related Research ${ }^{\mathbb{B}}$ editors and board members are on file with the publication and can be viewed on request.

Each author certifies that his or her institution approved the human protocol for this investigation, that all investigations were conducted in conformity with ethical principles of research, and that informed consent for participation in the study was obtained.

I. H. Rhyou, J.-H. Lee ( $₫)$, K. C. Kim, K. B. Ahn, S. C. Moon, H. J. Kim, J. H. Lee

Department of Orthopedic Surgery, Upper Extremity and

Microsurgery Center, Pohang SM Christianity Hospital, Daedo

Dong 94-5, Nam Gu, Pohang, Kyeongbuk, South Korea

e-mail: jiholee22@gmail.com
Methods Between June 2007 and June 2012, 100 patients with elbow fractures were included in the cohort, with 46 of these patients being excluded owing to incongruity for our surgical indication. Finally, 54 patients with surgically treated elbow fractures who had MRI preoperatively were assessed retrospectively. There were 17 elbows with isolated coronoid fractures, 22 with isolated radial head fractures, and 15 with combined coronoid and radial head fractures. Collateral ligament injury pattern and existence of distal humerus bone contusion were reviewed on MR images.

Results Patients with isolated radial head fractures were at greater risk of medial collateral ligament rupture compared with patients with isolated coronoid fractures (radial head only: 15 of 22 [68\%]; coronoid only: three of 17 [18\%]; odds ratio [OR], 10.0; 95\% CI, 2.2-46.5; p = 0.002). Patients with isolated coronoid fractures had greater risk of lateral ulnar collateral ligament ruptures (coronoid: 16 of 17 [94\%]; radial head: seven of 22 [32\%]; OR, 3.5; 95\% CI, 3.8-333.3; $\mathrm{p}<0.001)$. The presence of radial head fractures was associated with the risk of lateral bone bruising (isolated radial head fracture: 32 of 37 [86\%], isolated coronoid fracture: four of 17 [24\%]; OR, 29.6; 95\% CI, 5.2-168.9; $\mathrm{p}<0.001)$. Medial bone bruising was only detected in isolated coronoid fractures (isolated coronoid fracture: 12 of 17 [71\%], others: zero of 37 [0\%]). All isolated coronoid fractures involved the anteromedial facet of the coronoid (17 of 17; 100\%). However, combined coronoid and radial head fractures often involved the tip (13 of $15 ; 87 \%)$.

Conclusions Isolated coronoid fractures mostly involved the anteromedial facet of the coronoid process associated with lateral ulnar collateral ligament rupture and medial bone bruising. However, isolated radial head fractures were associated with medial collateral ligament rupture and 
lateral bone bruising. Combined coronoid and radial head fractures mostly involved a tip fracture of the coronoid with lateral ulnar collateral ligament rupture and lateral bone bruising. Thus surgeons may predict which ligament they should be aware of in the surgical field.

Level of evidence Level III, prognostic study.

\section{Introduction}

One of the most important factors that maintains normal function of the elbow is stability. It depends on osseous structural integrity and soft tissue constraints [9]. Loss of elbow stability can result in various bony structure derangements and ligamentous injuries. Before performing a stabilization procedure for an unstable elbow, it is important to determine the location of the main injuries and decide which structures to repair [3]. Coronoid, radial head, and combined coronoid and radial head fractures are common elbow fractures $[2,12,16]$. They are closely associated with ligamentous conditions [3, 8, 9, 15]. Anteromedial facet fractures of the coronoid process and isolated radial head fractures are known to be associated with the lateral ulnar collateral ligament $[5,6,9,13]$. However, some authors have asserted that comminuted isolated radial head fractures have a greater incidence of complete medial collateral ligament rupture $[1,5,13]$. Because the medial collateral ligament and radial head are primary and secondary valgus stabilizers of the elbow, complete rupture of the medial collateral ligament and a concomitant radial head fracture can induce severe disruption of elbow stability. Therefore, it is important for patients to undergo MRI to verify the ligamentous integrity before determining the treatment option (surgical versus conservative). However, MRI findings showing ligament injury with a concurrent radial head fracture have been rarely reported [5, 6, 13]. Although two studies have reported osseous injuries with ligamentous disruptions, studies describing existing conditions such as physical examination, surgical impressions, and demographic data are limited $[12,16]$. To the best of our knowledge, no study has compared various elbow fractures considering the osseous injury mechanism related to ligament status.

Therefore, the objectives of this study were: (1) to determine what ligamentous injury patterns (medial or lateral collateral) and bone contusion patterns (medial or lateral) are associated with isolated coronoid, isolated radial head, and combined coronoid and radial head fractures; (2) to correlate the osseous injury mechanism based on these findings with isolated coronoid, isolated radial head, and combined coronoid and radial head fractures; and (3) to determine whether isolated and combined coronoid fractures have different fracture lines through the coronoid (tip or anteromedial facet), speculated to be caused by different injury mechanisms.

\section{Patients and Methods}

This retrospective study was approved by our institutional review board. We evaluated a total of 100 patients (30 coronoid fractures, 55 radial head fractures, and 25 combined coronoid process and radial head fractures) who visited the emergency room of a single trauma center between June 2007 and June 2012. Three-dimensional (3D) CT scans were performed for all patients. However, MRI was performed for only 54 patients, who were appropriated for our surgical indications to coronoid and radial head fractures. The surgical indication for coronoid fractures was a fracture size of the coronoid process of 5 $\mathrm{mm}$ or more based on the study by Pollock et al. [11], or fracture classification of the coronoid fracture was O'Driscoll Type II or greater [9]. Radial neck fractures were surgically treated when a blocking or clicking sensation was felt during careful forearm rotation before surgery or the fracture was involved in one-third of the radius of the radial head with $2 \mathrm{~mm}$ displacement or more than $30^{\circ}$ angulation of the radial neck [13]. Ultimately, 54 patients were enrolled in this study ( 34 males and 20 females). The patients had a mean age of 39 years (range, 13-73 years). There were 17 patients with isolated coronoid fractures (15 males and two females), with a mean age of 42 years (range, 26-69 years); 22 patients with isolated radial head fractures (11 males and 11 females), with a mean age of 36 years (range, 18-52 years); and 15 patients with combined coronoid and radial head fractures (eight males and seven females), with a mean age of 41 years (range, 13-73 years) (Fig. 1).

Elbow dislocation was diagnosed based on initial radiographs and medical history obtained in the emergency department. Among all patients with combined coronoid and radial head fractures, dislocation was seen on initial radiographs of three (terrible triad).

Three-dimensional reconstructions were made from Digital Communications in Medicine (DICOM) files after 0.64-mm slice thickness CT scans were obtained. MR images were performed with 1.5-T (Intera; Philips, Amsterdam, The Netherlands; and Signa ${ }^{\mathrm{TM}}$ HDx, GE Healthcare, Milwaukee, WI, USA) or 3.0-T (MAGNETOM $^{\circledR}$ Verio; Siemens, Berlin, Germany) scanners. All MR images were performed within 1 week after trauma. Although T1- and T2-weighted image sequences were not uniform because each MRI scanner has different sequences, all scans were performed in $30^{\circ}$ to $60^{\circ}$ flexion allowed 


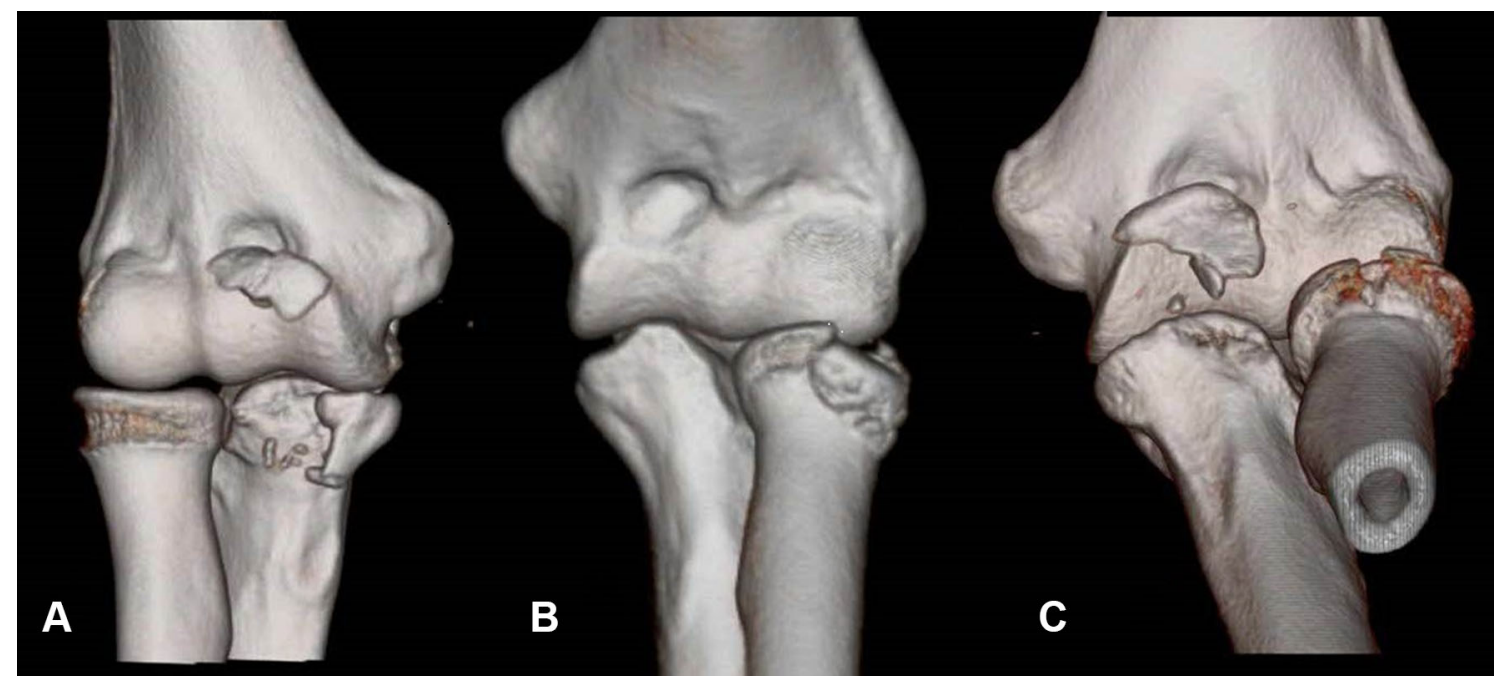

Fig. 1A-C (A) An isolated coronoid fracture, $(\mathbf{B})$ an isolated radial head fracture, and $(\mathbf{C})$ a combined coronoid and radial head fracture are shown.

by the degree of pain using the long axis of the humeral shaft as a datum line. Coronal, sagittal, and axial plane images were obtained.

\section{Three-dimensional CT and MRI Interpretation}

Radiographs and 3-D CT and MR images were interpreted by the two authors (J-HL and IHR) who had completed fellowships in shoulder and elbow surgery and had more than 5 years of elbow surgery experience, respectively. Postoperative radiographs and patient information were not provided for review. Structural characteristics of these fractures were analyzed by 3-D CT based on the classification systems of O'Driscoll et al. [9] and Charalambous et al. [1] for coronoid and radial head fractures, respectively.

Elbow dislocations developed in four patients with isolated coronoid fractures. Three of these dislocations were of the posteromedial type. One elbow dislocation was of the pure posterior type (the distal humerus was perched over the coronoid process) with an anteromedial facet and tip fracture of the coronoid process [9]. Three elbow dislocations occurred in the combined coronoid and radial head fracture group (terrible triad) (14\%), including two of the posterolateral type and one of the posteromedial type.

Collateral ligament injuries were divided into complete rupture, partial rupture, and intact subtypes. Complete rupture of the medial collateral ligament was classified as disappearance of continuity or translation on the medial condyle of the humerus or sublime tubercle of the ulna. Complete rupture of the lateral ulnar collateral ligament was classified as disappearance of continuity or translation on the posterior half of the lateral condyle of the humerus or crista supinatoris (lateral ulnar collateral ligament insertion), while partial rupture of the medial or lateral ulnar collateral ligament was classified as partial disappearance of continuity or with increased signal intensity on a T2-weighted MR image.

A lateral bone contusion was classified as bone edema at the radial head or the posterior aspect of the capitellum (Fig. 2). A medial bone contusion was defined as bone edema at the medial trochlea or the medial aspect of the coronoid process (Fig. 3). The mechanism of occurrence of a lateral bone contusion has been thought to be pathologic forearm external rotation whereas a medial bone contusion has been thought to be attributable to varus force [15].

\section{Statistical Analysis}

All statistical analyses were performed using SPSS, Version 18.0 (SPSS Inc, Chicago, IL, USA). Interobserver reliabilities of the O'Driscoll et al. classification [9], Charalambous et al. classification [1], and MRI findings were measured by Pearson's correlation coefficient analysis. Intraobserver reliabilities were not checked. A chisquare test was used to detect differences in the collateral ligament (complete versus partial or intact) and bone contusions among isolated coronoid fractures, isolated radial head fractures, and combined coronoid and radial head fractures. A probability less than 0.05 was considered statistically significant.

Interobserver reliability values for the classifications of O'Driscoll et al. and Charalambous et al. were 0.92 (95\% CI, 0.84-1.0), and 0.93 (95\% CI, 0.86-1.0), respectively. 

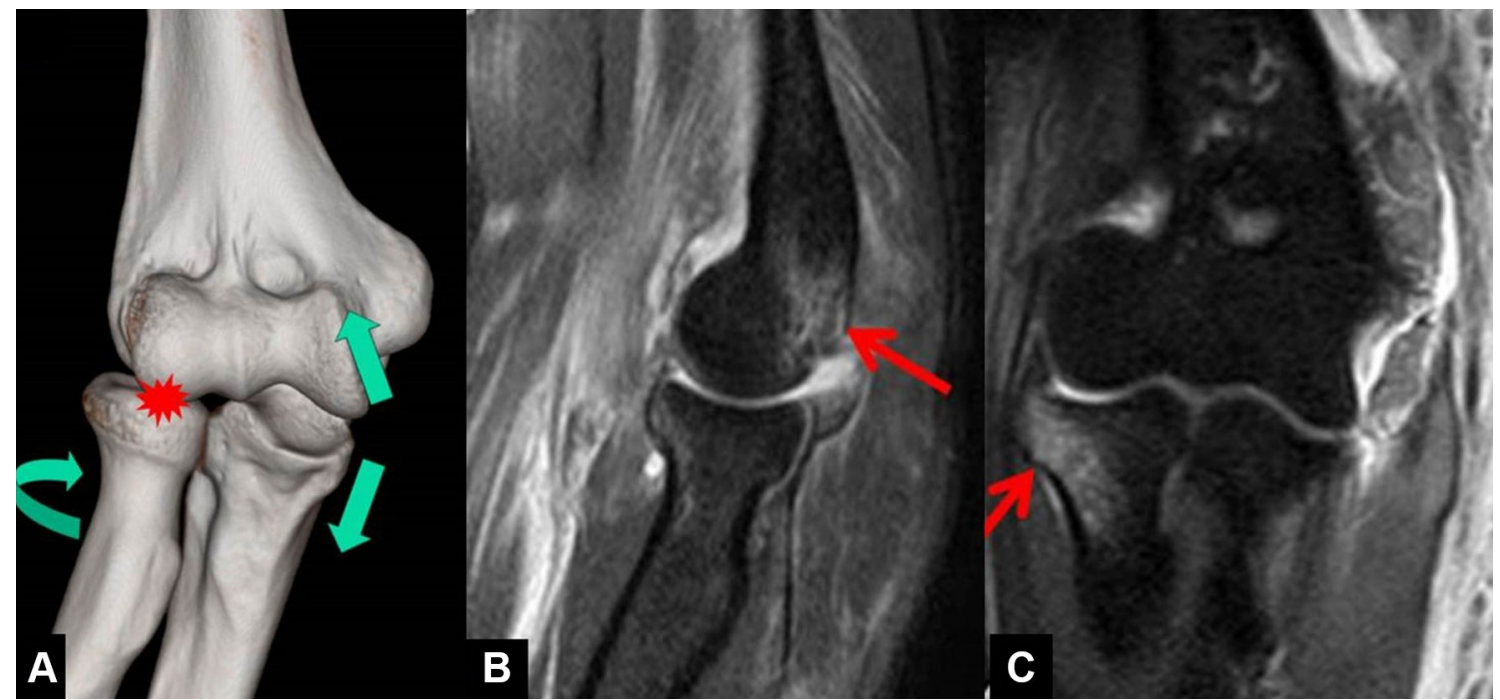

Fig. 2A-C (A) With isolated radial head fractures, valgus force leads to disengagement of the coronoid process from the trochlear notch and medial collateral ligament injury. Concomitant external rotation of the forearm can induce a posterior capitellar bone bruise and radial head fracture. The MR images show (B) a posterior capitellar bone bruise (arrow) and (C) a radial head bone bruise (arrow).

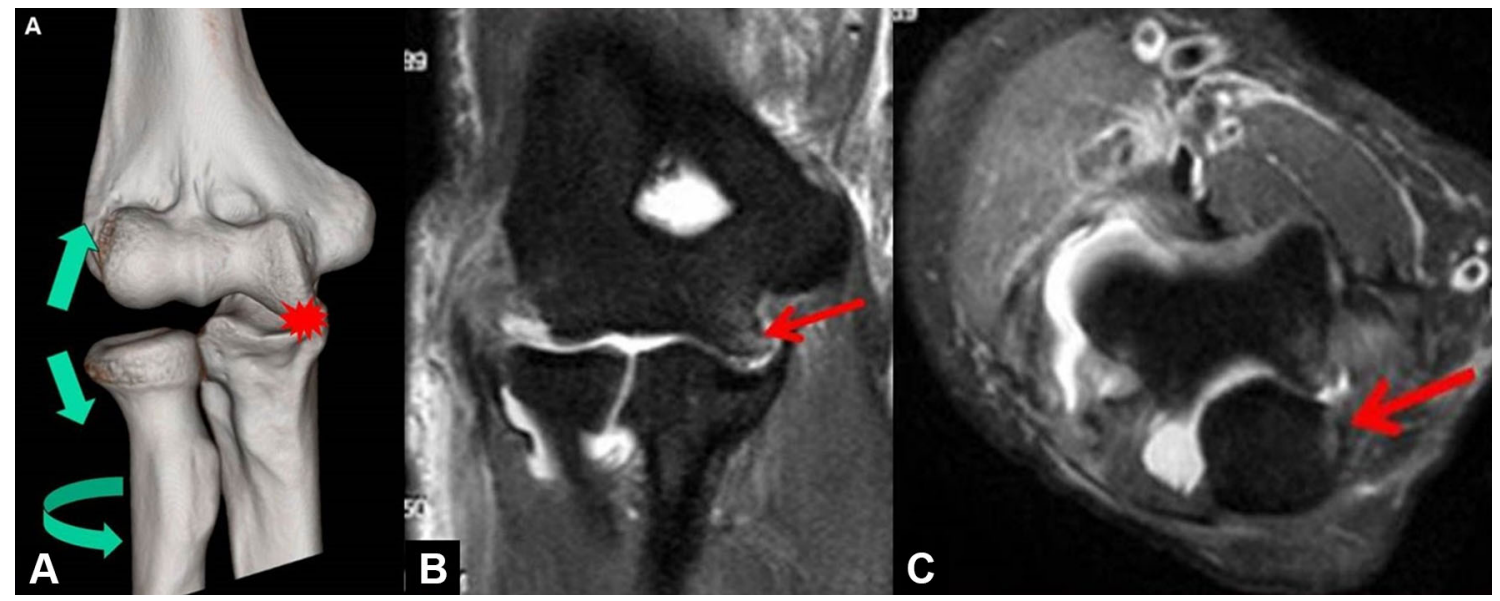

Fig. 3A-C The main mechanism of an isolated coronoid process fracture is shown. (A) Varus force leads to lateral ulnar collateral ligament injury and bony abutment of the coronoid process and trochlea, and concomitant forearm internal rotation force disengages

Those for collateral ligament status and appearance of bone edema by MRI findings were 0.80 (95\% CI, 0.67-0.92) and 0.92 (95\% CI, 0.84-1.0), respectively. MRI findings for complete ruptures of the medial collateral ligament were three of 17 elbows for isolated coronoid fractures, 15 of 22 elbows for isolated radial head fractures, and five of 15 elbows for combined coronoid and radial head fractures. Interobserver reliability values for complete rupture of the lateral ulnar collateral ligament were 16 of 17 elbows for isolated coronoid fractures, seven of 22 elbows for isolated radial head fractures, and 10 of 15 elbows for combined coronoid and radial head fractures. Lateral bone contusions the elbow. The MR images show a bony contusion $(\mathbf{B})$ of the medial side of the distal humerus (arrow), and $(\mathbf{C})$ of the coronoid process (arrow).

were seen in four of 17 elbows with isolated coronoid fractures, 19 of 22 with isolated radial head fractures, and 13 of 15 with combined coronoid and radial head fractures. Medial bone contusions were only detected in 12 of 17 elbows with isolated coronoid fractures (Table 1).

\section{Results}

Patients with isolated radial head fractures were at greater risk of medial collateral ligament rupture compared with patients with isolated coronoid fractures (radial head only: 
Table 1. Numbers of patients in isolated coronoid and isolated radial head fracture groups based on MRI findings and results of chi-square test and odds ratio

\begin{tabular}{|c|c|c|c|c|}
\hline Parameter & $\begin{array}{l}\text { Isolated } \\
\text { coronoid } \\
(\mathrm{n}=17)\end{array}$ & $\begin{array}{l}\text { Isolated } \\
\text { radial head } \\
(\mathrm{n}=22)\end{array}$ & $\begin{array}{l}\text { Odds ratio } \\
(95 \% \mathrm{CI})\end{array}$ & $\mathrm{p}$ Value \\
\hline Medial collateral ligament complete rupture & $3(17.6 \%)$ & $15(68.2 \%)$ & $34.5(3.8-333.3)$ & $<0.001$ \\
\hline Lateral ulnar collateral ligament complete rupture & $16(94.1 \%)$ & $7(31.8 \%)$ & $10(2.6-46.5)$ & 0.002 \\
\hline Lateral bone contusion & $4(17.6 \%)$ & $19(86.4 \%)$ & $29.6(5.2-168.9)$ & $<0.001$ \\
\hline Medial bone contusion & $12(70.6 \%)$ & 0 & N/A & N/A \\
\hline
\end{tabular}

$\mathrm{N} / \mathrm{A}=$ not applicable.

15 of 22 [68\%], coronoid only: three of 17 [18\%]; odds ratio $[\mathrm{OR}], 10.0 ; 95 \% \mathrm{CI}, 2.2-46.5 ; \mathrm{p}=0.002)$. Patients with coronoid fractures had greater risk of lateral ulnar collateral ligament ruptures (coronoid: 16 of 17 [94\%], radial head: seven of 22 [32\%]; OR, 3.5; 95\% CI, 3.8333.3; $\mathrm{p}<0.001$ ) (Table 1). The risk of complete rupture of the medial collateral ligament was greater with isolated radial head fractures than with combined coronoid and radial head fractures (radial head only: 15 of 22 [68\%], combined coronoid and radial head: five of 15 [33\%]; OR, $4.3 ; 95 \% \mathrm{CI}, 1.1-17.2 ; \mathrm{p}=0.037)$. The presence of radial head fractures increased the risk of lateral bone bruising (radial head fracture: 32 of 37 [86\%], isolated coronoid fracture: four of 17 [24\%]; relative risk, 29.9; 95\% CI, 6.3142.6; $\mathrm{p}<0.001)$. Stratified lateral bone bruising was associated with isolated radial head fractures (19 of 22; $86 \%$ ) and combined coronoid and radial head injuries (13 of $15 ; 87 \%)$. Medial bone bruising was only associated with isolated coronoid fractures (12 of 17 [71\%], others: zero of 37) (Fig. 4). All isolated coronoid fractures involved the anteromedial facet of the coronoid (17 of 17). However, combined injuries often involved the tip (13 of $15)$, with an anterolateral oblique pattern (Table 2).

\section{Discussion}

Collateral ligament injuries often are associated with coronoid and radial head fractures. However, the osseous injury mechanism related to ligament status remains unclear.

Although some studies have tried to establish the correlation between isolated coronoid fracture or isolated radial head fracture and ligamentous lesion [5, 6, 11, 13], there have been no published studies, to our knowledge, comparing isolated coronoid, isolated radial head, and combined coronoid and radial head fractures with ligamentous injuries and bony contusion in terms of injury mechanism.

Therefore, the objective of this study was to determine the association of fracture pattern with ligamentous injuries and bony contusion. In addition, coronoid fracture pattern was characterized in this study based on elbow fracture location.

Several limitations of this study should be mentioned. First, 46 patients were excluded owing to lack of MR images. However, in this study, because we focused on patients who underwent surgery among those with various elbow fractures, we only checked MR images of the patients who had surgery and had thorough application of our operative indication. Second, sensitivity and specificity testing of MRI readings for ligamentous injuries were not performed. In addition, intraobserver reliability was not obtained. Finally, elbow dislocation was not diagnosed if we did not have radiographs included in the hospital records that showed a dislocation. Fortunately, all patients with a history of dislocation mentioned in the emergency department records had initial radiographs showing the dislocation. However, combined coronoid and radial head fractures had only three terrible triad cases. Two of the three cases were posterolateral dislocations typically seen in patients with the terrible triad. We think possibly a higher proportion of patients had terrible triad fractures than we saw on initial radiographs, but the patients arrived through the emergency room and self-reduction may have occurred in some cases.

The incidence of collateral ligament injuries associated with coronoid fractures and/or radial head fractures is relatively high. Doornberg and Ring [4] reviewed 18 cases of coronoid anteromedial facet fractures and found that 15 of 18 patients sustained a lateral ulnar collateral ligament complex injury. van Riet et al. [16] presented a description of 333 cases of radial head fractures, of which 44 had a concurrent ligamentous injury. Isolated coronoid fractures likely occurred through varus stress with the posteromedial rotatory component, whereas isolated radial head fractures had valgus stress with forced forearm external rotation. Combined coronoid and radial head fractures also likely developed by valgus stress and external rotation force similar to isolated radial head fractures. However, discernable differences between isolated radial head fractures 


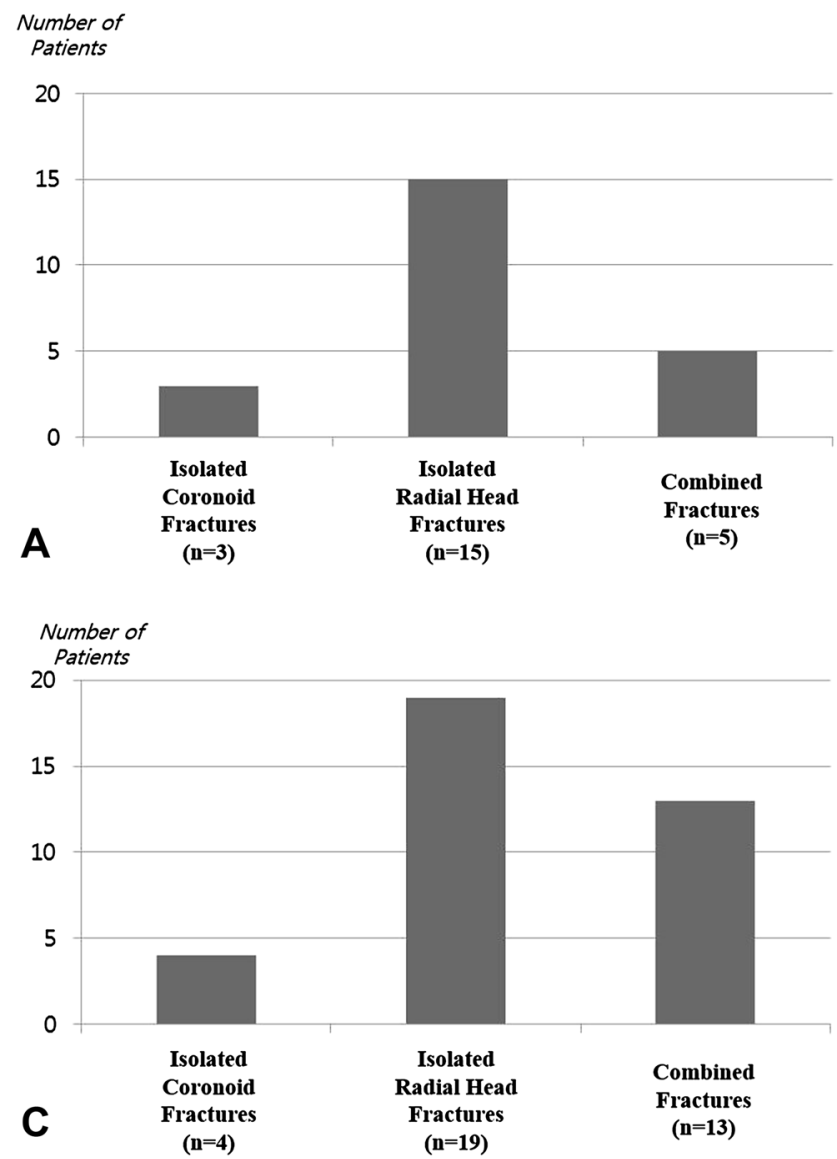

Fig. 4A-D (A) Patients with isolated radial head fractures were at greater risk of medial collateral ligament rupture compared with patients with isolated coronoid fractures (odds ratio [OR], 10.0; $95 \%$ CI, 2.2-46.5; p = 0.002), while the risk of complete rupture of the medial collateral ligament was greater for isolated radial head fractures than combined coronoid and radial head fractures $(\mathrm{OR}, 4.3$; $95 \% \mathrm{CI}, 1.1-17.2 ; \mathrm{p}=0.037)$. (B) Patients with isolated coronoid fractures had a greater risk for lateral ulnar collateral ligament

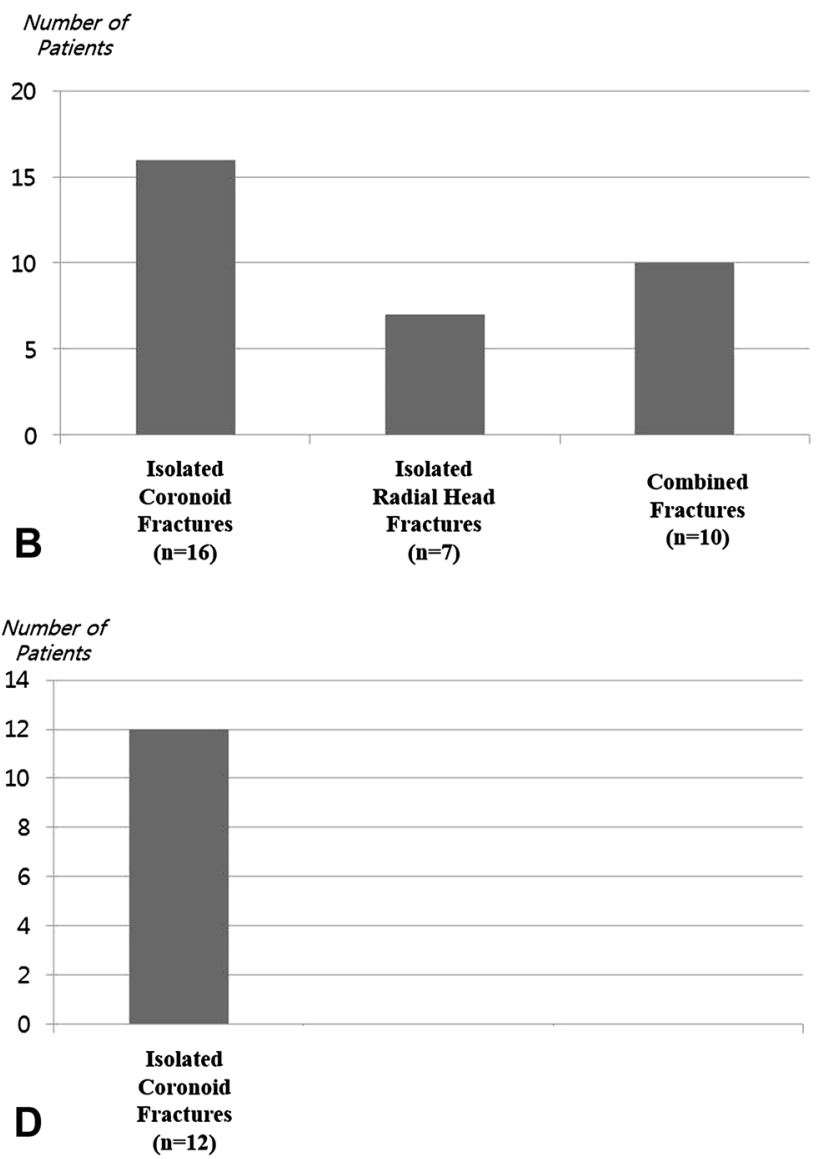

ruptures than for isolated radial head fractures (OR, 3.5; 95\% CI, 3.8 333.3; $\mathrm{p}<0.001)$. (C) Lateral bone contusions were more frequent in isolated radial head and combined coronoid and radial head fractures than in isolated coronoid fractures (radial head fracture: 32 of 37 [86\%], isolated coronoid fracture: four of 17 [24\%]; OR, 29.9; 95\% CI, 6.3-142.6; $\mathrm{p}<0.001$ ). (D) Medial bone contusions were detected at a high frequency $(63.2 \%)$ only in isolated coronoid fractures.

Table 2. Numbers of patients with isolated coronoid process fractures and combined fractures to specific site of coronoid fractures

\begin{tabular}{|c|c|c|c|c|}
\hline Parameter & $\begin{array}{l}\text { Isolated } \\
\text { coronoid fractures } \\
(\mathrm{n}=17)\end{array}$ & $\begin{array}{l}\text { Coronoid with } \\
\text { radial head fractures } \\
(\mathrm{n}=15)\end{array}$ & $\begin{array}{l}\text { Odds ratio } \\
(95 \% \mathrm{CI})\end{array}$ & $\mathrm{p}$ Value \\
\hline Anteromedial facet & $17(100 \%)$ & $1(6.7 \%)$ & $46.7(4.9-415.2)$ & $<0.001$ \\
\hline Tip & $0(0 \%)$ & $13(86.7 \%)$ & N/A & $<0.001$ \\
\hline Basal & $0(0 \%)$ & $1(6.7 \%)$ & N/A & N/A \\
\hline
\end{tabular}

N/A = not applicable.

and combined coronoid and radial head fractures are ratios of medial collateral ligament and lateral ulnar collateral ligament complete rupture (Table 3). Combined fractures had $66.7 \%$ of lateral ulnar collateral ligament injuries whereas isolated radial head fractures had $31.8 \%$. In addition, medial collateral ligament complete ruptures in combined fractures were $33.3 \%$, whereas isolated radial head fractures were $68.2 \%$ (Table 3 ). This would mean that the ratio of external rotation force related to valgus force of combined coronoid and radial head fractures would be 
Table 3. Numbers of patients with isolated radial head fractures and combined coronoid and radial head fractures

\begin{tabular}{|c|c|c|c|c|}
\hline Parameter & $\begin{array}{l}\text { Isolated } \\
\text { radial head } \\
\text { fractures } \\
(\mathrm{n}=22)\end{array}$ & $\begin{array}{l}\text { Combined } \\
\text { fractures } \\
(\mathrm{n}=15)\end{array}$ & $\begin{array}{l}\text { Odds ratio } \\
(95 \% \mathrm{CI})\end{array}$ & $\mathrm{p}$ Value \\
\hline Medial collateral ligament complete rupture & $15(68.2 \%)$ & $5(33.3 \%)$ & $4.3(1.1-17.2)$ & 0.037 \\
\hline Lateral ulnar collateral ligament complete rupture & $7(31.8 \%)$ & $10(66.7 \%)$ & $5.9(1.4-25.2)$ & 0.162 \\
\hline Lateral bone contusion & $19(89.4 \%)$ & $13(86.7 \%)$ & $1.0(0.2-7.0)$ & 0.979 \\
\hline Medial bone contusion & 0 & 0 & N/A & N/A \\
\hline
\end{tabular}

$\mathrm{N} / \mathrm{A}=$ not applicable.

higher than isolated radial head fractures regardless of total amount of external force. In other words, combined fractures would develop mainly by rotational force similar to simple elbow dislocations [14], followed frequently by lateral ligament complete ruptures. Isolated radial head fractures likely developed mainly by valgus force, and medial collateral ligament complete ruptures followed.

In isolated fractures of either the radial head or the coronoid, the area of bony contusion was opposite the ligament injury (lateral ulnar collateral ligament with medial bone contusion, medial collateral ligament with lateral contusion). The lateral bone contusion may be caused mainly by impingement of the radial head into the posterior capitellum during forced forearm external rotation (Fig. 2), which was suggested as the initial step in posterior elbow dislocation by Osborne and Cotterill [10]. The medial bony contusion might have been caused by impingement of the medial trochlea and anteromedial portion of the coronoid by varus stress (Fig. 3) [14]. In combined fractures, only lateral bone contusion was detected similar to isolated radial head fractures, however, dissimilar to isolated radial head fractures, lateral ligament injury developed more frequently (Table 3). According to the mechanism of posterior elbow dislocation, forced forearm external rotation may lead to lateral bony contusion with a stripping injury of the lateral ulnar collateral ligament concomitantly. Therefore, this result also supports that combined fractures would develop mainly by external rotation force [14].

Isolated coronoid fractures, presumably from posteromedial rotation and varus, produced consistent fractures of the anteromedial facet, but combined fractures of the radial head are likely from forced forearm external rotation and valgus force, generating tip-only fractures (anterolateral oblique pattern) (Fig. 5). If external rotation force progresses further in combined fractures, a posterolateral elbow dislocation may develop (terrible triad) (Fig. 5). Mellema et al. [7] pointed out differences between isolated coronoid fractures and the terrible triad fracture pattern.

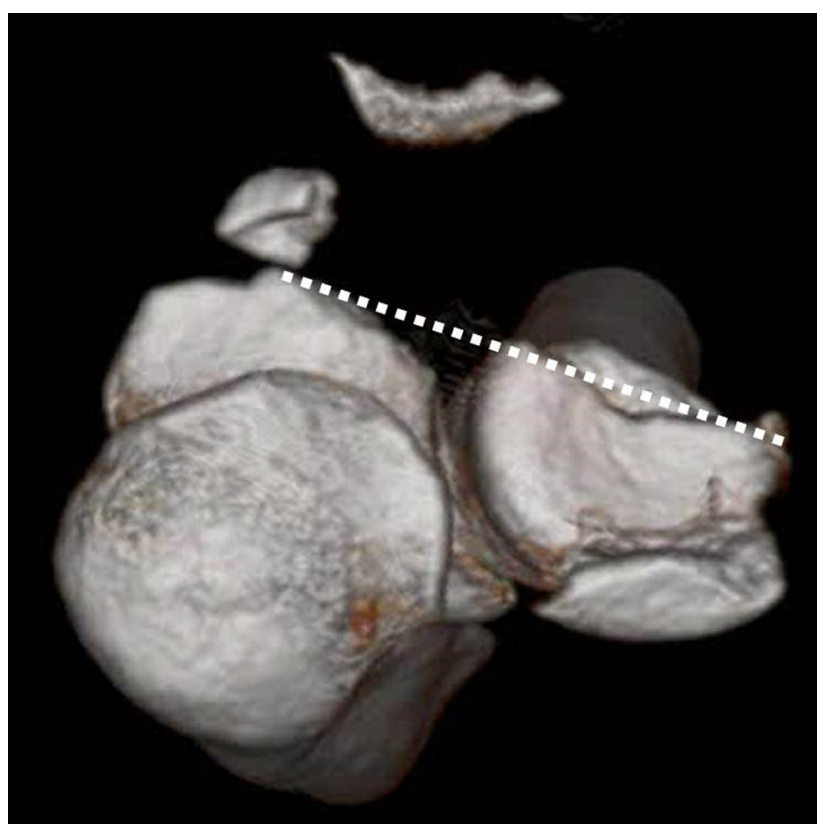

Fig. 5 With combined coronoid and radial head fractures, the fracture line of the coronoid process and radial head occurred in an extended line with the forearm fully supinated.

They used two-dimensional fracture mapping and heat mapping techniques to reveal the distribution of coronoid fracture lines among various fracture patterns. According to their maps, varus posteromedial rotational instability fractures were located on the relatively medial side and tip fractures associated with terrible triad injuries were laterally positioned.

Isolated coronoid fractures requiring surgery often involve the anteromedial facet of the coronoid process, related to posteromedial rotatory instability. The lateral ulnar collateral ligament is frequently ruptured but has fewer bone contusions on the posterior aspect of the capitellum; most of the bone bruising is found on the medial side of the distal humerus. Therefore, the surgeon should be aware of the status of the lateral ulnar collateral ligament during surgery. 
Isolated radial head fractures were associated with medial collateral ligament ruptures and lateral bone bruising. While the surgeon performs internal fixation or arthroplasty of the radial head fracture, the status of the medial collateral ligament should be confirmed.

Combined coronoid and radial head fractures mostly involved tip fractures of the coronoid with lateral ulnar collateral ligament rupture and lateral bone bruising, which occurred owing to axial loading, valgus, and forearm external rotation forces. If external rotation progresses further, a posterolateral elbow dislocation might develop.

\section{References}

1. Charalambous CP, Stanley JK, Mills SP, Hayton MJ, Hearnden A, Trail I, Gagey O. Comminuted radial head fractures: aspects of current management. J Shoulder Elbow Surg. 2011;20:996-1007.

2. Cohen MS. Fractures of the coronoid process. Hand Clin. 2004;20:443-453.

3. DiPaola M, Geissler WB, Osterman AL. Complex elbow instability. Hand Clin. 2008;24:39-52.

4. Doornberg JN, Ring DC. Fracture of the anteromedial facet of the coronoid process. J Bone Joint Surg Am. 2006;88:2216-2224.

5. Itamura J, Roidis N, Mirzayan R, Vaishnav S, Learch T, Shean C. Radial head fractures: MRI evaluation of associated injuries. $J$ Shoulder Elbow Surg. 2005;14:421-424.

6. Kaas L, van Riet RP, Turkenburg JL, Vroemen JP, van Dijk CN, Eygendaal D. Magnetic resonance imaging in radial head fractures: most associated injuries are not clinically relevant. $J$ Shoulder Elbow Surg. 2011;20:1282-1288.
7. Mellema JJ, Doornberg JN, Dyer GS, Ring D. Distribution of coronoid fracture lines by specific patterns of traumatic elbow instability. J Hand Surg Am. 2014;39:2041-2446.

8. Morrey BF, An KN. Stability of the elbow: osseous constraints. $J$ Shoulder Elbow Surg. 2005;14(1 suppl):174S-178S.

9. O’Driscoll SW, Jupiter JB, Cohen MS, Ring D, McKee MD. Difficult elbow fractures: pearls and pitfalls. Instr Course Lect. 2003;52:113-134.

10. Osborne G, Cotterill P. Recurrent dislocation of the elbow. $J$ Bone Joint Surg Br. 1966;48:340-346.

11. Pollock JW, Brownhill J, Ferreira L, McDonald CP, Johnson J, King G. The effect of anteromedial facet fractures of the coronoid and lateral ulnar collateral ligament injury on elbow stability and kinematics. J Bone Joint Surg Am. 2009;91:1448-1458.

12. Pugh DM, Wild LM, Schemitsch EH, King GJ, McKee MD. Standard surgical protocol to treat elbow dislocations with radial head and coronoid fractures. J Bone Joint Surg Am. 2004;86: $1122-1130$.

13. Rhyou IH, Kim KC, Kim KW, Lee JH, Kim SY. Collateral ligament injury in the displaced radial head and neck fracture: correlation with fracture morphology and management strategy to the torn ulnar collateral ligament. J Shoulder Elbow Surg. 2013;22:261-267.

14. Rhyou IH, Kim YS. New mechanism of the posterior elbow dislocation. Knee Surg Sports Traumatol Arthrosc. 2012;20: $2535-2541$.

15. Ring D, Jupiter JB, Zilberfarb J. Posterior dislocation of the elbow with fractures of the radial head and coronoid. J Bone Joint Surg Am. 2002;84:547-551.

16. van Riet RP, Morrey BF, O'Driscoll SW, van Glabbeek F. Associated injuries complicating radial head fractures: a demographic study. Clin Orthop Relat Res. 2005;441:351-355. 